\title{
Polynomials with zeros on systems of curves*
}

\author{
Vilmos Totik ${ }^{\dagger}$ \\ Dedicated to László Leindler on his 80th birthday
}

\begin{abstract}
On a compact subset of the complex plane the supremum norm of a polynomial of degree $n$ with leading coefficient 1 must be at least the $n$-th power of the logarithmic capacity of the set. In general, nothing more can be said, but if the polynomial also has zeros on the outer boundary, then those zeros may raise the minimal norm. The paper quantifies how much zeros on the boundary raise the norm on sets bounded by finitely many smooth Jordan curves. For example, $k_{n}$ zeros results in a factor $\left(1+c k_{n} / n\right)$, while $k_{n}$ excessive zeros on a subarc of the boundary compared to the expected value based on the equilibrium measure introduces an exponential factor $\exp \left(c k_{n}^{2} / n\right)$. The results are sharp, and they are related to Turán's power-sum method in number theory. It is also shown by an example that the smoothness condition cannot be entirely dropped.
\end{abstract}

\section{Introduction}

Let $C_{1}=\{z|| z \mid=1\}$ be the unit circle. It is immediate from the maximum principle that if $P_{n}(z)=z^{n}+\cdots$ is a so called monic polynomial i.e. with leading coefficient 1 , then the supremum of $\left|P_{n}(z)\right|$ on the unit circle is at least 1 (apply the maximum principle to $z^{n} P_{n}(1 / z)$ ), which we write in the form $\left\|P_{n}\right\|_{C_{1}} \geq 1$. It is also relatively easy to see that if such a polynomial has a zero somewhere on the unit circle, then $\left\|P_{n}\right\|_{C_{1}} \geq 1+1 / 30 n$ (instead of $1+1 / 30 n$ the best lower bound was determined in [6]). On the other hand, G. Halász [5] showed that for every $n$ there is a monic polynomial $Q_{n}(z)=z^{n}+\cdots$ with a zero at 1 and of norm $\left\|Q_{n}\right\|_{C_{1}} \leq \exp (2 / n)$. These results are related to Turán's power sum method in number theory.

The paper [11] discussed what happens if more than one zero is on $C_{1}$. It was shown that if $P_{n}$ has $k_{n}$ zeros on $C_{1}$, then $\left\|P_{n}\right\|_{C_{1}} \geq 1+c k_{n} / n$ with a universal $c>0$. Furthermore, if $P_{n}$ has at least $k_{n}+n|J| / 2 \pi$ zeros on a subarc $J$ of $\Gamma$, then

$$
\left\|P_{n}\right\|_{\Gamma} \geq \exp \left(c k_{n}^{2} / n\right)
$$

*AMS Classification 42C05, 31A15, Keywords: monic polynomials, minimal norm, zeros on the boundary, equilibrium measure

†Supported by the Europearn Research Council Advanced Grant No. 267055 
again with some universal constant $c>0$ (here $|J|$ denotes the arc length of $J$ ). This second result is sharp: it follows from Halász' theorem mentioned before that if $z_{1, n}, \ldots, z_{k_{n}, n}$ are arbitrary $k_{n} \leq n / 2$ points on the unit circle, then there is a $P_{n}(z)=z^{n}+\cdots$ such that $P_{n}$ vanishes at each $z_{j, n}$, and $\left\|P_{n}\right\|_{C_{1}} \leq$ $\exp \left(4 k_{n}^{2} / n\right)$. The sharpness of the first result is also true: it was proved by Andrievskii and Blatt [2] that if $\alpha>1$ and $z_{1, n}, \ldots, z_{k_{n}, n}$ are $k_{n}$ points on the unit circle such that any two of them are of distance $\geq \alpha 2 \pi / n$, then there is a polynomial $P_{n}(z)=z^{n}+\cdots$ such that $P_{n}$ vanishes at each $z_{j, n}$, and $\left\|P_{n}\right\|_{C_{1}} \leq$ $1+D_{\alpha} k_{n} / n$ where $D_{\alpha}$ is a constant that depends only on $\alpha$. Note that this is not true for $\alpha<1$. Indeed, if $\alpha<1$, then consider the $\alpha 2 \pi / n$-spaced sequence $X_{n}$ of $k_{n}$ points consisting of

$$
e^{i j \alpha 2 \pi / n}, \quad j=0,1, \ldots, k_{n}-1,
$$

and let $J=J_{n}$ be the (counterclockwise) arc on the unit circle from 1 to $e^{i k_{n} \alpha 2 \pi / n}$. Now if $P_{n}(z)=z^{n}+\cdots$ is a polynomial such that it has a zero at every point of $X_{n}$, then there are $\geq(1-\alpha) k_{n}$ excess zeros of $P_{n}$ on $J_{n}$ compared to $n\left|J_{n}\right| / 2 \pi$. Therefore, it follows from (1) that

$$
\left\|P_{n}\right\|_{C_{1}} \geq \exp \left(c(1-\alpha)^{2} k_{n}^{2} / n\right),
$$

which is much bigger than $1+D_{\alpha} k_{n} / n$ if $k_{n} \rightarrow \infty$.

In the present paper we prove similar results for monic polynomials on unions of finitely many Jordan curves. We note that [11] used heavily the circular symmetry of $C_{1}$, in particular some results on trigonometric polynomials, so the method of [11] is not applicable here, and we need a totally new approach.

To formulate our results we need some basic notions from logarithmic potential theory, see [3], [4] or [9] for the necessary concepts. In particular, $\operatorname{cap}(K)$ denotes the logarithmic capacity of a compact set $K \subset \mathbf{C}$, and $\mu_{K}$ denotes its equilibrium measure (in the cases we are going to discuss this $\mu_{K}$ exists). Furthermore, $\|\cdot\|_{K}$ denotes supremum norm on $K$.

Recall (see [9, Theorem 5.5.4]) that if $K$ is a compact set with logarithmic capacity $\operatorname{cap}(K)$ and $P_{n}(z)=z^{n}+\cdots$ is a monic polynomial of degree $n$, then

$$
\left\|P_{n}\right\|_{K} \geq \operatorname{cap}(K)^{n} .
$$

In general, nothing more can be said, for if $K=T_{m}^{-1}\left(C_{1}\right)$ is the complete inverse image of $C_{1}$ under some monic polynomial $T_{m}$ of degree $m$, then the equality in (2) holds for all $P_{n}=T_{m}^{k}, n=m k, k=1,2, \ldots$.

Now we show that if $K$ consists of finitely many smooth Jordan curves, then zeros on $K$ raise the norm compared to the theoretically possible minimum $\operatorname{cap}(K)^{n}$.

Theorem 1.1 Let $\Gamma$ be a finite system of $C^{1+\alpha}, \alpha>0$, smooth Jordan curves lying exterior to one another. If $P_{n}$ is a monic polynomial of degree $n$ that has $k_{n}$ zeros on $\Gamma$, then $\left\|P_{n}\right\|_{\Gamma} \geq\left(1+c k_{n} / n\right) \operatorname{cap}(\Gamma)^{n}$ with a $c$ that depends only on $\Gamma$. 
Recall that a Jordan curve is the homeomorphic image of a circle, while a Jordan arc is the homeomorphic image of a segment.

In the theorem, and in what follows, the $C^{1+\alpha}$-smoothness could be replaced by Dini-smoothness (see [8, Sec. 3.3]) of the derivatives of the (arc-length) parametrization functions of the individual components of $\Gamma$.

The theorem is true if $\Gamma$ has arc components, but for a completely different reason. Indeed, if $\Gamma$ contains a Jordan arc, then there is a $\beta>0$ such that for all monic polynomials we have $\left\|P_{n}\right\|_{\Gamma} \geq(1+\beta) \operatorname{cap}(\Gamma)^{n}$, see [14, Theorem 1].

When there are more than one components, Theorem 1.1 is interesting only for certain $n$ 's, since then there is a $\beta>0$ and a subsequence $\mathcal{N}$ of the natural numbers such that $\left\|P_{n}\right\|_{\Gamma} \geq(1+\beta) \operatorname{cap}(\Gamma)^{n}$ for all $P_{n}$ and $n \in \mathcal{N}$ (see [14, Theorem 2]).

An example for the application of Theorem 1.1 is the case of Fekete polynomials. If $K \subset \mathbf{C}$ is a compact set, then $n$-th Fekete points for $K$ maximize the product

$$
\prod_{1 \leq i<j \leq n}\left|z_{j, n}-z_{i, n}\right|
$$

among all $n$-touples $\left\{z_{1, n}, \ldots, z_{n, n}\right\} \subset K$. These Fekete points necessarily lie on the outer boundary of $K$ (which is the boundary of the unbounded component of $\overline{\mathbf{C}} \backslash K)$, so if this outer boundary consists of finitely many $C^{1+\alpha}$-smooth Jordan curves or arcs, then we necessarily have for the Fekete polynomials $P_{n}(z)=\prod_{1}^{n}\left(z-z_{j, n}\right)$ the bound

$$
\left\|P_{n}\right\|_{K} \geq(1+\beta) \operatorname{cap}(K)^{n}, \quad n=1,2, \ldots
$$

with some $\beta>0$. Indeed, if there are only Jordan curves on the outer boundary then this follows from by Theorem 1.1, while if there are arc components, as well, then, as we have just mentioned, the statement follows from [14, Theorem $1]$.

Next, we show that if on a subarc of $\Gamma$ a $P_{n}$ has too many zeros compared to the "expected number" relative to the equilibrium measure, then the norm of $P_{n}$ is considerably larger than the theoretical lower bound $\operatorname{cap}(\Gamma)^{n}$.

Theorem 1.2 Let $\Gamma$ be a system of $C^{2+\alpha}, \alpha>0$, Jordan curves lying exterior to one another. If $P_{n}$ is a monic polynomial of degree $n$ that has at least $k_{n}+$ $n \mu_{\Gamma}(J)$ zeros on a subarc $J$ of $\Gamma$, then $\left\|P_{n}\right\|_{\Gamma} \geq \exp \left(c k_{n}^{2} / n\right) \operatorname{cap}(\Gamma)^{n}$ with a $c$ that depends only on $\Gamma$ and $z_{0}$.

If we apply this to a subarc $J$ of $\Gamma$ and to all the subarcs that build up the complement $\Gamma \backslash J$, then we obtain

Corollary 1.3 Let $\Gamma$ be a system of $C^{2+\alpha}, \alpha>0$, Jordan curves lying exterior to one another, let $P_{n}$ be a monic polynomial of degree $n$ that has all its zeros on $\Gamma$, and let $\nu_{P_{n}}$ denote the normalized counting measure on the zeros of $P_{n}$. Then uniformly in subarcs $J$ of $\Gamma$ we have

$$
\left|\nu_{P_{n}}(J)-\mu_{\Gamma}(J)\right| \leq C \sqrt{\frac{\log \left(\left\|P_{n}\right\|_{\Gamma} / \operatorname{cap}(\Gamma)^{n}\right)}{n}} .
$$


For one curve this corollary is not new, it follows from a theorem of Andrievskii and Blatt, see [1, Theorem 3.4.1].

Theorem 1.2 is actually true if $\Gamma$ consists of Jordan curves and arcs. Indeed, the claim when $J$ lies on a curve component of $\Gamma$ can be handled as we shall do it in the proof of Theorem 1.2. On the other hand, if $J$ lies on an arc component of $\Gamma$, then we can use the result from [1, Theorem 2.4.2], according to which we have

$$
\frac{k_{n}}{n} \leq C \sqrt{\frac{\log \left(\left\|P_{n}\right\|_{\Gamma} / \operatorname{cap}(\Gamma)^{n}\right)}{n}} .
$$

We have already mentioned that both Theorems 1.1 and 1.2 are best possible when $\Gamma=C_{1}$, so one cannot expect any better estimate then what these theorems claim. But actually, more is true, e.g. if $\Gamma$ consists of a single smooth Jordan curve, and if $z_{1, n}, \ldots, z_{k_{n}, n}$ are arbitrary $k_{n} \leq n / 2$ points on $\Gamma$, then there is a $P_{n}(z)=z^{n}+\cdots$ such that $P_{n}$ vanishes at each $z_{j, n}$, and $\left\|P_{n}\right\|_{\Gamma} \leq \exp \left(C k_{n}^{2} / n\right)$ with a constant $C$ that depends only on $\Gamma$. We shall not prove this statement, it can be derived from Halász' result mentioned before.

Theorem 1.1 shows that if all zeros of $P_{n}(z)=z^{n}+\cdots$ are on $\Gamma$, and $\Gamma$ has the required smoothness, then $(3)$ is true, i.e. in this case the ratio $\left\|P_{n}\right\|_{\Gamma} / \operatorname{cap}(\Gamma)^{n}$ stays away from 1 , it cannot approach the theoretical minimal value 1 . It is somewhat surprising that for this conclusion one needs some kind of smoothness.

Theorem 1.4 There is a Jordan curve $\Gamma$, a sequence $\mathcal{N}$ of the natural numbers, and for all $n \in \mathcal{N}$ a monic polynomial $Q_{n}(z)=z^{n}+\cdots$ of degree $n$ such that $Q_{n}$ has all its zeros on $\Gamma$, and still

$$
\lim _{n \rightarrow \infty, n \in \mathcal{N}} \frac{\left\|Q_{n}\right\|_{\Gamma}}{\operatorname{cap}(\Gamma)^{n}}=1
$$

\section{Proofs of Theorem 1.1 and 1.2}

Proof of Theorem 1.1. We follow the proof of [12, Theorem 1], and we shall use from [12] the following lemma (see [12, Lemma 2.2]):

Lemma 2.1 There are $\delta, \theta>0$ depending only on $\Gamma$ such that if $J=\widehat{a b}$ is a subarc of $\Gamma$ of length at most $\delta$ and if a polynomial $P_{n}$ of degree at most $n$ has at least $\theta n|J|$ zeros on $J$, then $\left|P_{n}(b)\right| \leq 1 / 3\left\|P_{n}\right\|_{\Gamma}$.

It is folklore (see e.g. [13, Proposition 2.2]) that on $\Gamma$ the equilibrium measure is absolutely continuous with respect to arc measure with continuous density, and so there is a constant $C_{0}$ such that for all $\operatorname{arcs} I$ on $\Gamma$ we have

$$
|I| \leq C_{0} \mu_{\Gamma}(I) .
$$

Let now $P_{n}$ be the polynomial from Theorem 1.1. If $\left\|P_{n}\right\|_{\Gamma} \geq(3 / 2) \operatorname{cap}(\Gamma)^{n}$, then we are ready. Otherwise, consider the set $H \subset \Gamma$ of those $z$ on $\Gamma$ for 
which $\left|P_{n}(z)\right| \leq\left\|P_{n}\right\|_{\Gamma} / 2$. This set consists of arcs, say $J_{1}, \ldots, J_{j}, \ldots$, on which $\left|P_{n}(z)\right| \leq(3 / 4) \operatorname{cap}(\Gamma)^{n}$. Next, we claim that

$$
n \log \operatorname{cap}(\Gamma) \leq \int \log \left|P_{n}\right| d \mu_{\Gamma}=\int_{H}+\int_{\Gamma \backslash H}=I_{1}+I_{2} .
$$

Indeed, from properties of equilibrium measures (see e.g. [10, (I.4.8)]) it follows that

$$
\int \log |z-t| d \mu_{\Gamma}(z)= \begin{cases}\log \operatorname{cap}(\Gamma) & \text { if } z \text { lies inside } \Gamma \\ \log \operatorname{cap}(\Gamma)+g_{\mathbf{C} \backslash \Gamma}(z, \infty) & \text { otherwise, }\end{cases}
$$

where $g_{\mathbf{C} \backslash \Gamma}(z, \infty)$ denotes the Green's function of the unbounded component of $\mathbf{C} \backslash \Gamma$ with pole at infinity. Hence the left-hand side is always at least as large as $\log \operatorname{cap}(\Gamma)$, which proves the inequality in (6) if we write $\log \left|P_{n}(z)\right|$ in the form $\sum_{j} \log \left|z-z_{j}\right|$ with the zeros of $P_{n}$ for $z_{j}$.

Now

$$
I_{2} \leq \mu_{\Gamma}(\Gamma \backslash H) \log \left\|P_{n}\right\|_{\Gamma},
$$

and for any $j$

$$
I_{1} \leq \mu_{\Gamma}(H) \log \left((3 / 4) \operatorname{cap}(\Gamma)^{n}\right) \leq \mu_{\Gamma}(H) n \log \operatorname{cap}(\Gamma)+\mu_{\Gamma}\left(J_{j}\right) \log (3 / 4) .
$$

These, $\mu_{\Gamma}(\Gamma \backslash H)+\mu_{\Gamma}(H)=1$ and (6) yield the theorem if one of the $J_{j}$ 's is of length bigger than $\delta$ (with the $\delta$ from Lemma 2.1), for then its harmonic measure $\mu_{\Gamma}\left(I_{j}\right)$ is at least $\delta_{1}$ with some $\delta_{1}>0$ that depends only on $\Gamma$.

If, on the other hand, all $J_{j}$ have length at most $\delta$, then, by Lemma 2.1, the number of zeros of $P_{n}$ on $J_{j}$ is at most $\theta n\left|J_{j}\right|$ with the $\theta$ from Lemma 2.1, since the value of $P_{n}$ at the endpoints of $J_{j}$ is $\left\|P_{n}\right\|_{\Gamma} / 2$. Therefore, using also (5), we have with some $C_{0}$

$$
k_{n} \leq \theta n \sum_{j}\left|J_{j}\right| \leq \theta n C_{0} \sum \mu_{\Gamma}\left(J_{j}\right)=\theta n C_{0} \mu_{\Gamma}(H),
$$

and so from we obtain from (6) and (8)-(9)

$$
\begin{aligned}
\mu_{\Gamma}(\Gamma \backslash H) \log \left\|P_{n}\right\| & \geq I_{2} \geq n \log \operatorname{cap}(\Gamma)-I_{1} \\
& \geq n \log \operatorname{cap}(\Gamma)-\mu_{\Gamma}(H) \log \left((3 / 4) \operatorname{cap}(\Gamma)^{n}\right) \\
& \geq \mu_{\Gamma}(\Gamma \backslash H) n \log \operatorname{cap}(\Gamma)+\left(-\log (3 / 4) / \theta C_{0}\right) k_{n} / n,
\end{aligned}
$$

and this completes the proof.

In the rest of the paper we shall need the concept of the logarithmic potential of a measure $\nu$ :

$$
U^{\nu}(z):=\int \log \frac{1}{|z-t|} d \nu(t)
$$


In particular, we get from (7) for the equilibrium potential (in the cases we consider)

$$
U^{\mu_{\Gamma}}(z)=\log \frac{1}{\operatorname{cap}(\Gamma)}, \quad z \in \Gamma,
$$

while if $\nu$ is the counting measure of a polynomial, then

$$
U^{\nu}(z)=-\log \left|P_{n}(z)\right| .
$$

Proof of of Theorem 1.2. We mention first of all, that for a single Jordan curve Theorem 1.2 can be easily deduced from [1, Theorem 4.1.1] by taking the balayage of the normalized zero counting measure $\nu_{n}$ onto $\Gamma$ (see the discussion below). In the general case we proceed similarly, but we shall need to prove the analogue of [1, Theorem 4.1.1].

Let $\nu_{n}$ be the normalized counting measure on the zeros of $P_{n}$ and let $\tilde{\nu}_{n}$ be the measure that we obtain by taking the balayage of $\nu_{n}$ out of each component of $\mathbf{C} \backslash \Gamma$ (one by one, in any order). Since taking the balayage out of a bounded region does not change the logarithmic potential on the boundary, while taking balayage out of an unbounded region increases it by a positive constant on the boundary (see Theorems [10, Theorems II.4.1, II. 4.4]), it follows that

$$
U^{\tilde{\nu}_{n}}(z) \geq U^{\nu_{n}}(z)=-\frac{1}{n} \log \left|P_{n}(z)\right|, \quad z \in \Gamma .
$$

Therefore, for the measure $\sigma=\mu_{\Gamma}-\tilde{\nu}_{n}$ we have for $z \in \Gamma$

$$
U^{\sigma}(z) \leq U^{\mu_{\Gamma}}(z)+\frac{1}{n} \log \left|P_{n}(z)\right| \leq \log \frac{\left\|P_{n}\right\|_{\Gamma}^{1 / n}}{\operatorname{cap}(\Gamma)}
$$

(recall that, by (11) we have $U^{\mu_{\Gamma}}(z)=\log 1 / \operatorname{cap}(\Gamma)$ on $\left.\Gamma\right)$. Now we can deduce the claim from the following discrepancy theorem.

Theorem 2.2 Let $\Gamma$ be a system of $C^{2+\alpha}, \alpha>0$, Jordan curves lying exterior to one another, and let $\sigma=\sigma^{+}-\sigma^{-}$be a signed measure on $\Gamma$ with the properties that $\sigma(\Gamma)=0, \sigma^{+} \leq L \mu_{\Gamma}$ with some constant $L$, and with some constant a

$$
U^{\sigma}(z) \leq a, \quad z \in \Gamma .
$$

Then there is a constant $M$ depending only on $L$ and $\Gamma$ such that for any subarc $J$ of $\Gamma$ we have $|\sigma(J)| \leq M \sqrt{a}$.

The proof of this theorem will be given in the next section, but first let us see how it proves Theorem 1.2. By the assumption we have

$$
\sigma(J)=\left(\mu_{\Gamma}-\tilde{\nu}_{n}\right)(J) \leq\left(\mu_{\Gamma}-\nu_{n}\right)(J) \leq-\frac{k_{n}}{n} .
$$

On the other hand by (12) and Theorem 2.2

$$
|\sigma(J)| \leq M \sqrt{\log \frac{\left\|P_{n}\right\|_{\Gamma}^{1 / n}}{\operatorname{cap}(\Gamma)}} .
$$


Hence

$$
\log \frac{\left\|P_{n}\right\|_{\Gamma}^{1 / n}}{\operatorname{cap}(\Gamma)} \geq c \frac{k_{n}^{2}}{n^{2}}
$$

and the claim follows.

\section{Proof of Theorem 2.2}

By the principle of domination (see [10, Theorem II.3.2]) the inequality (13) holds for all $z \in \mathbf{C}$. Therefore, for a single Jordan curve this theorem is a special case of the one-sided discrepancy theorem [1, Theorem 4.1.1]. Unfortunately, the proof of [1, Theorem 4.1.1] is quite involved and uses conformal maps of the inner and outer domains onto the unit circle in such an essential way that one cannot claim that the proof goes over to the case when several components are present. Still we use the ideas of that proof adapted to our needs.

As we have just mentioned, we may assume (13) to hold for all $z \in \mathbf{C}$. We may also assume that in the $C^{2+\alpha}$-smoothness of $\Gamma$ the parameter $\alpha$ lies in between 0 and 1 (in other words, we do not allow $\alpha$ to be 1 ).

Let $\Gamma_{0}, \Gamma_{1}, \ldots, \Gamma_{k}$ be the components of $\Gamma$ (each being a $C^{2+\alpha}$ Jordan curve) and assume that $\Gamma_{0}$ contains the arc $J$. Let $D_{j}^{-}$resp. $D_{j}^{+}$be the bounded resp. unbounded connected component of $\mathbf{C} \backslash \Gamma_{j}$ and $\Omega$ the unbounded component of $\mathbf{C} \backslash \Gamma$. Then $\Omega=\cap_{j} D_{j}^{+}$, and the connected components of $\mathbf{C} \backslash \Gamma$ are $\Omega, D_{0}^{-}, \ldots, D_{k}^{-}$.

In the proof of Theorem 2.2 below $s=s_{\Gamma}$ denotes the arc length measure on $\Gamma$ and we set

$$
\delta=\sqrt{a}, \quad r=\delta^{2}=a,
$$

and we may assume $a$ so small that the arcs of length $\sim \delta$ to be constructed below all exist (indeed, since $|\sigma(J)| \leq \sigma^{+}(\Gamma)+\sigma^{-}(\Gamma)$, the statement in the theorem follows with some $M$ for $a \geq a_{0}$ if $a_{0}$ is some fixed number).

We may also assume that the length of $J$ is at most half of the length of $\Gamma_{0}$. Attach a subarc of $\Gamma$ of length $\delta$ to $J$ at both endpoints to form the arc $J_{\delta}$. Let $f_{0}$ be a $C^{2}$ function (with respect to arc length) on $\Gamma$ such that $f_{0}=0$ on all components $\Gamma_{j}$ except for $j=0, f_{0}(z)=0$ for $z \notin J_{2 \delta}, f_{0}(z)=1$ for $z \in J_{\delta}$, and on the two arcs of $J_{2 \delta} \backslash J_{\delta}$ we have $0 \leq f_{0} \leq 1,\left|d f_{0} / d s\right| \leq C / \delta$, $\left|d^{2} f_{0} / d s^{2}\right| \leq C / \delta^{2}$ with some $C$ depending only on $\Gamma$. The existence of such and $f_{0}$ is clear (it is easy to construct such a function on the unit circle and then map it onto $\Gamma_{0}$ ). Solve now the Dirichlet problem with this boundary function on all components of $\mathbf{C} \backslash \Gamma$. Let the solution in $\cup_{j} D_{j}^{-}$be $f_{-}$and the solution in $\Omega$ be denoted by $f_{+}$. Of course, for $j>0$ in $D_{j}^{-}$we solve then the Dirichlet problem with zero boundary function, so in $\cup_{j=1}^{k} D_{j}^{-}$the function $f_{0}$ is identically 0 . In any case $0 \leq f_{ \pm} \leq 1$ everywhere.

First we claim the following smoothness for this function. 
Lemma 3.1 Let $z \in D_{0}^{-}, t \in \Gamma_{0}$ and assume that $|z-t| \leq 3 r$. Then

$$
\left|f_{-}(z)-f_{0}(t)\right| \leq C \delta .
$$

The same is true if $z \in \Omega$ :

$$
\left|f_{+}(z)-f_{0}(t)\right| \leq C \delta .
$$

For $z \in D_{j}^{-}, t \in \Gamma_{j}, j \geq 1$ the corresponding estimate is trivial since $f_{-}(z)=$ $f_{0}(t)=0$, and finally for $z \in \Omega$ and $t \in \Gamma_{j}, j \geq 1$

$$
\left|f_{+}(z)-f_{0}(t)\right|=f_{+}(z) \leq C r \leq C \delta,
$$

(all under the assumption $|z-t| \leq 3 r$ ).

Proof. Let $\Phi$ be a conformal map from $D_{0}^{-}$onto the unit disk. Then $\Phi$ extends continuously to the boundary $\Gamma_{0}$ of $D_{0}^{-}$and by the Kellogg-Warschawski

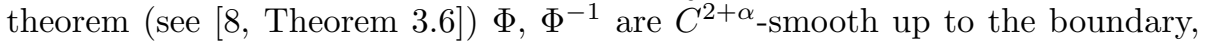
and their derivatives vanish nowhere, including the boundary (see $[8$, Theorem $3.5]$ ). We may assume that $x=\Phi(z)$ lies on $[1 / 2,1]$ (recall that $r=a$ is small). We verify that with $\varphi(w)=f_{-}\left(\Phi^{-1}(w)\right)$ we have

$$
|\varphi(x)-\varphi(1)| \leq C \delta .
$$

This will prove (15) since on the boundary $C_{1}$ of the unit disk we have $\left|d \varphi\left(e^{i u}\right) / d u\right| \leq$ $C / \delta$ because $\left|d f_{0} / d s\right| \leq C / \delta$, and the distance of $x$ and $\Phi(t)$, and hence that of 1 and $\Phi(t)$, is less than $C r$, so

$$
\begin{aligned}
\left|f_{-}(z)-f_{0}(t)\right| & =|\varphi(x)-\varphi(\Phi(t))| \leq|\varphi(x)-\varphi(1)|+|\varphi(1)-\varphi(\Phi(t))| \\
& \leq C \delta+C r / \delta \leq C \delta
\end{aligned}
$$

since $r=\delta^{2}$.

In (18) we have $1-x \leq C r$ and by Poisson's formula

$$
\begin{aligned}
\varphi(x)-\varphi(1) & =\frac{1}{2 \pi} \int_{-\pi}^{\pi}\left(\varphi\left(e^{i u}\right)-\varphi(1)\right) \frac{1-x^{2}}{1-2 x \cos u+x^{2}} d u \\
& =: \quad \int_{-\pi}^{\pi}\left(\varphi\left(e^{i u}\right)-\varphi(1)\right) P_{x}(u) d u .
\end{aligned}
$$

Since

$$
1-2 x \cos u+x^{2}=(1-x)^{2}+4 x \sin ^{2} \frac{u}{2},
$$

the integral over $|u| \geq \delta$ is at most

$$
C \int_{|u| \geq \delta} \frac{1-x}{u^{2}} d u \leq C \frac{1-x}{\delta} \leq C \frac{r}{\delta} \leq C \delta .
$$

We write in the integral over $|u| \leq \delta$

$$
\varphi\left(e^{i u}\right)-\varphi(1)=B u+B(u) \frac{u^{2}}{\delta^{2}},
$$


where $B$ is a constant and $B(u)$ is a function with $|B(u)| \leq C$ (this follows from the fact that $\left|d^{2} f_{0} / d s^{2}\right| \leq C / \delta^{2}$ and that $\Phi$ and its inverse are $C^{2+\alpha}$ functions). Now, by symmetry, the integral of $B u P_{x}(u)$ on $|u| \leq \delta$ vanishes, so we are left with estimating

$$
\int_{|u| \leq \delta} \frac{u^{2}}{\delta^{2}} P_{x}(u) d u
$$

for which the bound $C(1-x) / \delta \leq C r / \delta=C \delta$ immediately follows since $P_{x}(u) \leq$ $1 /(1-x)$ for $|u| \leq 1-x$ and $P_{x}(u) \leq(1-x) / u^{2}$ for $|u| \geq 1-x$. This proves (15).

As for (16), let now $\Phi$ be the conformal map of the unbounded domain $D_{0}^{+}$ onto the unit disk. Then $\varphi(w)=f_{+}\left(\Phi^{-1}(w)\right)$ is harmonic in a fixed annulus $A:=\{z|\rho \leq| z \mid<1\}$ (note that this function is not defined everywhere in the unit disk since $f_{+}$is not defined in the inner domains $D_{j}^{-}$). Now follow the preceding proof, just replace the Poisson kernel $P_{x}(u)$ with the density $\tilde{P}_{x}(1, u)$ and $\tilde{P}_{x}(\rho, u)$ on $C_{1}$ and on $\{z|| z \mid=\rho\}$, resp., of the harmonic measure on this annulus with respect to the point $x$. We have

$\varphi(x)-\varphi(1)=\int_{-\pi}^{\pi}\left(\varphi\left(e^{i u}\right)-\varphi(1)\right) \tilde{P}_{x}(1, u) d u+\int_{-\pi}^{\pi}\left(\varphi\left(\rho e^{i u}\right)-\varphi(1)\right) \tilde{P}_{x}(\rho, u) d u$.

Using the symmetry of $\tilde{P}_{x}(1, u)$ and the fact that $\tilde{P}_{x}(1, u) \leq P_{x}(u)$ (which follows from the monotonicity of the harmonic measure in the domain) the first integral can be handled exactly as above and we get the bound $C \delta$ for it. In estimating the second integral in (19), let $\omega(z, J, G)$ denote the harmonic measure in a domain $G$ of a boundary arc $J \subset \partial G$ with respect to a point $z \in G$. To complete the proof of (16) it is sufficient to show for estimating the second integral in (19) that $\omega\left(x, C_{1}, A\right) \geq 1-C r$. Indeed, then we get the bound

$$
C \int_{-\pi}^{\pi} \tilde{P}_{x}(\rho, u) d u=C\left(1-\int_{-\pi}^{\pi} \tilde{P}_{x}(1, u) d u\right)=C\left(1-\omega\left(x, C_{1}, A\right)\right) \leq C r \leq C \delta
$$

for that second integral in (19). But $\omega\left(x, C_{1}, A\right) \geq 1-C r$ is clear, since

$$
\omega\left(x, C_{1}, A\right)=\frac{\log x / \rho}{\log 1 / \rho} \geq 1-C(1-x) \geq 1-C r .
$$

Finally, (17) follows similarly. Indeed, first we note that (use the monotonicity of harmonic measures in the domain) $\omega\left(z, \Gamma_{0}, \Omega\right) \leq \omega\left(z, \gamma_{j}, \Omega_{j}^{*}\right)$ where $\gamma_{j}$ is a fixed level curve $\left\{\zeta|| \Psi_{j}(\zeta) \mid=1+b\right\}$ of the conformal map $\Psi_{j}$ from the outer domain $D_{j}^{+}$onto the exterior of the unit disk and $\Omega_{j}^{*}$ is the domain enclosed by $\gamma_{j}$ and $\Gamma_{j}$ (take such a level curve which goes close to $\Gamma_{j}$ not intersecting any other $\left.\Gamma_{s}\right)$. Now $\Psi_{j}$ maps $\Omega_{j}^{*}$ into the annulus $A^{*}:=\{w|1<| w \mid<1+b\}$ for which the harmonic measure is

$$
\omega\left(\Psi_{j}(z),\{z|| z \mid=1+b\}, A^{*}\right)=\frac{\log \left|\Psi_{j}(z)\right|}{\log (1+b)} \leq C\left(\left|\Psi_{j}(z)\right|-1\right) \leq C r,
$$


and so, by the conformal invariance of harmonic measures, we have

$$
\omega\left(z, \Gamma_{0}, \Omega\right) \leq \omega\left(z, \gamma_{j}, \Omega_{j}^{*}\right)=\omega\left(\Psi_{j}(z),\{z|| z \mid=1+b\}, A^{*}\right) \leq C r .
$$

Hence,

$$
f_{+}(z)=\int f_{0} d \omega(z, \cdot, \Omega) \leq \int_{\Gamma_{0}} d \omega(z, \cdot, \Omega)=\omega\left(z, \Gamma_{0}, \Omega\right) \leq C r
$$

As special case we get that if $z \in D_{0}^{-}$and $\operatorname{dist}(z, J) \leq r$, then

$$
0 \leq 1-f_{-}(z) \leq C \delta \text {. }
$$

The same is true if $z \in \Omega$ and $\operatorname{dist}(z, J) \leq r$ :

$$
0 \leq 1-f_{+}(z) \leq C \delta \text {. }
$$

For $\operatorname{dist}\left(z, \Gamma_{0} \backslash J_{3 \delta}\right) \leq r$ the corresponding estimates are

$$
0 \leq f_{-}(z) \leq C \delta \quad \text { resp. } \quad 0 \leq f_{+}(z) \leq C \delta .
$$

Let $\tilde{f}$ be the function that agrees with $f_{-}$in $\cup_{j} D_{j}^{-}$and with $f_{+}$in $\Omega$, and, as in the proof of [1, Theorem 4.1.1], set

$$
f(z)=\frac{1}{r^{2}} \int \tilde{f}(u) K\left(\frac{z-u}{r}\right) d m(u),
$$

where $m$ is the two-dimensional Lebesgue-measure and $K$ is a circular symmetric nonnegative $C^{\infty}$ kernel function with support in the unit disk and with integral 1: $\int K d m=1$. Since $\tilde{f}$ is harmonic in each component of $\mathbf{C} \backslash \Gamma$, it follows that for $\operatorname{dist}(z, \Gamma)>2 r$ we have $\tilde{f}(z)=f(z)$. So for such $z$ the function $f$ is again harmonic in a neighborhood of $z$, and therefore $\Delta f(z)=0$, where $\Delta$ denotes the Laplacian.

Recall now Green's formula

$$
\int_{H}(v \Delta u-u \Delta v) d m=\int_{\partial H}\left(v \frac{\partial u}{\partial \mathbf{n}}-u \frac{\partial v}{\partial \mathbf{n}}\right) d s_{\partial H}
$$

where $H$ is a domain with $C^{2}$ boundary, $s_{\partial H}$ is the arc measure on $\partial H$ and $\mathbf{n}$ denotes the inner normal at a boundary point. With $v=1$ and $u=f$ we conclude

$$
\int_{\partial H} \frac{\partial f}{\partial \mathbf{n}} d s_{\partial H}=0
$$

for any domain $H$ (with $C^{2}$-smooth boundary) on which $f$ is harmonic. Let $\gamma_{j}$, $j=0, \ldots, k$, be $C^{2}$ curves lying in $D_{j}^{-}$of distance $>2 r$ from $\Gamma_{j}$, let $H_{j}$ be the domain enclosed by $\gamma_{j}$ and let $\mathbf{n}_{j}$ denote the inner normal to a generic point on 
the boundary $\gamma_{j}$ of $H_{j}$. In addition, let $\gamma^{*}$ be a $C^{2}$-curve in $\Omega$ enclosing $\Gamma$ which is of distance $>2 r$ from $\Gamma$, let $H^{*}$ be the exterior domain to $\gamma^{*}$, and denote $\mathbf{n}^{*}$ the inner normal to the boundary $\gamma^{*}$ of $H^{*}$ at a generic point of $\gamma^{*}$. Finally, let $H$ be the domain enclosed by $\gamma^{*}$ and the curves $\gamma_{j}, j=0,1, \ldots, k$, and let $\mathbf{n}$ be the inner normal at a generic point of $\partial H$. By (24) we have

$$
\int_{\gamma_{j}} \frac{\partial f}{\partial \mathbf{n}} d s_{\partial H}=\int_{\partial H_{j}}\left(-\frac{\partial f}{\partial \mathbf{n}_{j}}\right) d s_{\partial H_{j}}=-\int_{\partial H_{j}} \frac{\partial f}{\partial \mathbf{n}_{j}} d s_{\partial H_{j}}=0,
$$

and since a similar relation holds for $\gamma^{*}$ (using the outer domain $H^{*}$ where $f$ is harmonic including the point infinity), it follows that

$$
\int_{\partial H} \frac{\partial f}{\partial \mathbf{n}} d s_{\partial H}=0
$$

Therefore, Green's formula with $u=f$ and $v=1$ yields

$$
\int_{H} \Delta f d m=0
$$

and since $\Delta f=0$ outside $H$, it also follows that

$$
\int_{\mathbf{C}} \Delta f d m=0 .
$$

Next, we use Green's formula in these domains with $u=f$ and $v=\log |\zeta-Z|$ where $Z \in \Gamma$ is an arbitrary fixed point. Since both $f(\zeta)$ and $\log |\zeta-Z|$ are harmonic in each $H_{j}$, it follows that

$$
\int_{\partial H_{j}}\left(v \frac{\partial f}{\partial \mathbf{n}_{j}}-f \frac{\partial v}{\partial \mathbf{n}_{j}}\right) d s_{\partial H_{j}}=0 .
$$

For $H^{*}$ the formula is different: let $H^{* *}$ be the intersection of $H^{*}$ with the interior of a large circle $C_{R}$ about $Z$ of radius $R$. Then Green's formula for $H^{* *}$ gives

$$
\left(\int_{\partial H^{*}}+\int_{C_{R}}\right)\left(v \frac{\partial f}{\partial \mathbf{n}^{* *}}-f \frac{\partial v}{\partial \mathbf{n}^{* *}}\right) d s_{\partial H^{* *}}=0 .
$$

Now on $C_{R}$ we have $v=R, \partial v / \partial \mathbf{n}^{* *}=-1 / R$, while $f(\zeta)=f(\infty)+o(1)$ as $R \rightarrow \infty$, hence it follows that

$$
\int_{\partial H^{*}}\left(v \frac{\partial f}{\partial \mathbf{n}^{* *}}-f \frac{\partial v}{\partial \mathbf{n}^{* *}}\right) d s_{\partial H^{*}}=-\log R \int_{C_{R}} \frac{\partial f}{\partial \mathbf{n}^{* *}} d s_{C_{R}}-2 \pi f(\infty)+o(1) .
$$

Finally, again from Green's formula applied in the outer domain of $C_{R}$ with $u=f$ and (this time with) $v=1$ we get (note that $f$ is harmonic in that outer domain including the point infinity)

$$
\int_{C_{R}} \frac{\partial f}{\partial \mathbf{n}^{* *}} d s_{C_{R}}=0
$$


All in all, we obtain for $R \rightarrow \infty$

$$
\int_{\partial H^{*}}\left(v \frac{\partial f}{\partial \mathbf{n}^{*}}-f \frac{\partial v}{\partial \mathbf{n}^{*}}\right) d s_{\partial H^{*}}=-2 \pi f(\infty) .
$$

(26) and (27) yield

$$
\int_{\partial H}\left(v \frac{\partial f}{\partial \mathbf{n}}-f \frac{\partial v}{\partial \mathbf{n}}\right) d s_{\partial H}=2 \pi f(\infty) .
$$

Next, let $Z \in \Gamma$ and $D_{\tau}$ a small disk around $Z$ of radius $\tau$. Using Green's formula in the domain $H \backslash D_{\tau}$ it follows from (28) that with $v(\zeta)=\log |\zeta-Z|$

$$
\begin{aligned}
\int_{H \backslash D_{\tau}}(f \Delta v-v \Delta f) d m & =\int_{\partial\left(H \backslash D_{\tau}\right)}\left(v \frac{\partial f}{\partial \mathbf{n}}-f \frac{\partial v}{\partial \mathbf{n}}\right) d s_{\partial H} \\
& =2 \pi f(\infty)+\int_{\partial D_{\tau}}\left(v \frac{\partial f}{\partial \mathbf{n}}-f \frac{\partial v}{\partial \mathbf{n}}\right) d s_{\partial D_{r}},
\end{aligned}
$$

where, in the last integral, $\mathbf{n}$ still points inside $H$. On $\partial D_{\tau}$ we have $\partial v / \partial \mathbf{n}=$ $1 / \tau$, so for $\tau \rightarrow 0$ we obtain

$$
\int_{H}(f \Delta v-v \Delta f) d m=2 \pi f(\infty)-2 \pi f(Z) .
$$

Finally, since $\Delta v=0$ everywhere but at $Z$, we conclude

$$
f(Z)-f(\infty)=\frac{1}{2 \pi} \int \log |\zeta-Z| \Delta f(\zeta) d m(\zeta) .
$$

Integrate this formula with respect to $d \sigma(Z)$ ! Noting that $\sigma(\mathbf{C})=\sigma(\Gamma)=0$, it follows that

$\int f d \sigma=\int_{\Gamma} \frac{1}{2 \pi} \int \log |\zeta-Z| \Delta f(\zeta) d m(\zeta) d \sigma(Z)=\frac{1}{2 \pi} \int\left(-U^{\sigma}(\zeta)\right) \Delta f(\zeta) d m(\zeta)$.

This and (25) give

$$
\int f d \sigma=\frac{1}{2 \pi} \int\left(a-U^{\sigma}(\zeta)\right) \Delta f(\zeta) d m(\zeta),
$$

where $a$ is the bound in Theorem 2.2. Using this form we shall below derive the following key statement:

$$
\left|\int f d \sigma\right| \leq C \delta
$$

Based on this inequality, we now complete the proof of Theorem 2.2 as follows.

$$
\begin{aligned}
-\sigma(J) & =-\int_{J} f d \sigma-\int_{J}(1-f) d \sigma \\
& =-\int f d \sigma-\int_{J}(1-f) d \sigma+\int_{J_{3 \delta} \backslash J} f d \sigma+\int_{\Gamma \backslash J_{3 \delta}} f d \sigma \\
& \leq\left|\int f d \sigma\right|+\int_{J}(1-f) d \sigma^{-}+\sigma^{+}\left(J_{3 \delta} \backslash J\right)+\int_{\Gamma \backslash J_{3 \delta}} f d \sigma^{+} .
\end{aligned}
$$


For the first term on the right we use (30), for the second one the estimate $0 \leq 1-f \leq C \delta$ (see (20) and (21) and the definition of $f$ ), for the third term the assumption in the theorem according to which $\sigma^{+}\left(J_{3 \delta} \backslash J\right) \leq L \mu_{\Gamma}\left(J_{3 \delta} \backslash J\right) \leq C \delta$, and finally for the last term we use (22) which gives $0 \leq f \leq C \delta$ on $\Gamma \backslash J_{3 \delta}$. All in all, we obtain $-\sigma(J) \leq C \delta$. On applying this with $J$ replaced by $\Gamma_{0} \backslash J$ (well, technically, represent here $\Gamma_{0} \backslash J$ as the union of two arcs with arc length smaller than half of the length of $\Gamma$ ) and by $\Gamma_{1}, \ldots, \Gamma_{k}$, respectively, and on using that $\sigma(\Gamma)=0$, we also get the reversed inequality $\sigma(J) \leq C \delta$, and the proof of Theorem 2.2 is complete pending the proof of (30).

Proof of (30). First we give an estimate on $\Delta f(\zeta)$. This is zero everywhere where $f$ is harmonic, so we only have to give a bound for it in the case when $\operatorname{dist}(\zeta, \Gamma) \leq 2 r$. Clearly

$$
\Delta f(\zeta)=\frac{1}{r^{2}} \Delta \int \tilde{f}(u) K\left(\frac{\zeta-u}{r}\right) d m(u)=\frac{1}{r^{2}} \int \tilde{f}(u) \Delta K\left(\frac{\zeta-u}{r}\right) d m(u) .
$$

Since $K$ vanishes outside the unit disk, Green's formula gives exactly as above

$$
\int \Delta K\left(\frac{\zeta-u}{r}\right) d m(u)=0
$$

and therefore

$$
\Delta f(\zeta)=\frac{1}{r^{2}} \int(\tilde{f}(u)-\tilde{f}(\zeta)) \Delta K\left(\frac{\zeta-u}{r}\right) d m(u)
$$

and here the kernel $K((\zeta-u) / r)$ vanishes unless $|\zeta-u| \leq r$. Therefore, in the non-vanishing case, both $\zeta$ and $u$ lie of distance $\leq 3 r$ from the same point $t \in \Gamma$ (which is the closest point on $\Gamma$ to $\zeta$ ), and hence Lemma 3.1 gives the bound $|f(u)-f(\zeta)| \leq C \delta$. On the other hand,

$$
\left|\Delta K\left(\frac{\zeta-u}{r}\right)\right| \leq \frac{C}{r^{2}}
$$

by the $C^{\infty}$ property of $K$, and therefore we obtain (recall that $r=\delta^{2}$ )

$$
|\Delta f(\zeta)| \leq \frac{C}{r^{2}} \int_{|u-\zeta| \leq r} \delta \frac{1}{r^{2}} d m(u) \leq \frac{C \delta}{r^{2}} \leq \frac{C}{r \delta}
$$

(When $z$ is close to a $\Gamma_{j}, j \geq 1$ then actually we can do even better, namely there $|\Delta f(\zeta)| \leq C / r$ holds by (17)). Now plug this into (29), and note that the integrand vanishes outside the set

$$
V_{r}:=\{z \mid \operatorname{dist}(z, \Gamma) \leq 2 r\},
$$

to obtain

$$
\left|\int f d \sigma\right| \leq \frac{1}{2 \pi} \int\left(a-U^{\sigma}(\zeta)\right)|\Delta f(\zeta)| d m(\zeta) \leq \frac{C}{r \delta} \int_{V_{r}}\left(a-U^{\sigma}(\zeta)\right) d m(\zeta) .
$$


We are going to show that here the integral on the the right-hand side is at most Car.

For some $\tau>0$ consider the set $[-\tau, \tau] \times \Gamma$, and the mapping $H(x, y)=$ $y+\mathbf{n}_{y} x$ from $[-\tau, \tau] \times \Gamma$ onto some subset $V$ of the complex plane, where $\mathbf{n}_{y}$ is the inner unit normal to the domain $\Omega$ at the point $y \in \Gamma$ (imagine moving a segment of length $2 \tau$ along $\Gamma$ in such a way that it is always perpendicular to $\Gamma$ and its center lies on $\Gamma$ ). For small but fixed $\tau$ the family of systems of curves $\Gamma_{x}:=\left\{y+\mathbf{n}_{y} x \mid y \in \Gamma\right\}, x \in[-\tau, \tau]$, are uniformly of $C^{1+\alpha}$ (see the Appendix at the end of the paper). Since for nonnegative continuous functions $F$ supported in $V$ we have

$$
\frac{1}{\Lambda} \int F d m \leq \int_{-\tau}^{\tau} \int_{\Gamma_{x}} F\left(y+\mathbf{n}_{y} x\right) d s_{\Gamma_{x}}\left(y+\mathbf{n}_{y} x\right) d x \leq \Lambda \int F d m,
$$

with some constant $\Lambda$ depending only on $\Gamma$, it follows that if we define the measure $m^{*}$ by the formula

$$
\int_{-\tau}^{\tau} \int_{\Gamma_{x}} F\left(y+\mathbf{n}_{y} x\right) d \mu_{\Gamma_{x}}\left(y+\mathbf{n}_{y} x\right) d x=\int F d m^{*},
$$

for all continuous $F$ supported in $V$, then $d m \sim d m^{*}$ in $V$ because

$$
d \mu_{\Gamma_{x}}\left(y+\mathbf{n}_{y} x\right) \sim d s_{\Gamma_{x}}\left(y+\mathbf{n}_{y} x\right)
$$

(here for measure $\mu, \nu$ the relation $\mu \sim \nu$ means that $\mu \leq C \nu$ and $\nu \leq C \mu$ with some constant $C$ ). Also, for some fixed $\alpha>0$ and all $0<r<\tau / \alpha$ the image $V_{r}^{*}$ of $[-\alpha r, \alpha r] \times \Gamma$ under the mapping $H$ covers the set $V_{r}$ from (31), therefore

$$
\begin{gathered}
\int_{V_{r}}\left(a-U^{\sigma}\right) d m \leq C_{0} \int_{V_{r}^{*}}\left(a-U^{\sigma}\right) d m^{*}=C_{0} a \int_{V_{r}^{*}} d m^{*}-C_{0} \int_{V_{r}^{*}} U^{\sigma} d m^{*} \\
\leq C_{1} a \int_{V_{r}^{*}} d m-C_{0} \int_{V_{r}^{*}} U^{\sigma} d m^{*} \leq C_{2} a r-C_{0} \int_{V_{r}^{*}} U^{\sigma} d m^{*} .
\end{gathered}
$$

For the last integral we have

$$
\int_{V_{r}^{*}} U^{\sigma} d m^{*}=\int_{-\alpha r}^{\alpha r} \int_{\Gamma_{x}} U^{\sigma}\left(y+\mathbf{n}_{y} x\right) d \mu_{\Gamma_{x}}\left(y+\mathbf{n}_{y} x\right) d x,
$$

and if we write here

$$
U^{\sigma}\left(y+\mathbf{n}_{y} x\right)=-\int_{\Gamma} \log \left|y+\mathbf{n}_{y} x-t\right| d \sigma(t)
$$

and switch the order of integration we can continue the preceding line as

$$
=\int_{-\alpha r}^{\alpha r} \int_{\Gamma} U^{\mu_{\Gamma x}}(t) d \sigma(t) d x .
$$

Since $\sigma$ has total mass 0 , this is the same as

$$
=\int_{-\alpha r}^{\alpha r}\left(\int_{\Gamma} U^{\mu_{\Gamma_{x}}}(t)+\log \operatorname{cap}\left(\Gamma_{x}\right)\right) d \sigma(t) d x
$$


Now

$$
U^{\mu_{\Gamma_{x}}}(u)+\log \operatorname{cap}\left(\Gamma_{x}\right)=0
$$

for $u \in \Gamma_{x}$ (see (7)), and from the uniform $C^{1+\alpha}$-smoothness of the curves $\Gamma_{x}$ we get along (34) that for $t \in \Gamma$ we have

$$
\left|U^{\mu_{\Gamma x}}(t)+\log \operatorname{cap}\left(\Gamma_{x}\right)\right| \leq C_{3}|x| .
$$

We shall prove (35) in the Appendix at the end of the paper.

Putting all these together we obtain (with $|\sigma|=\sigma^{+}+\sigma^{-}$)

$$
\left|\int_{V_{r}^{*}} U^{\sigma} d m^{*}\right| \leq C_{3}|\sigma|(\Gamma) \int_{-\alpha r}^{\alpha r}|x| d x \leq C_{4} r^{2}=C_{4} a r
$$

since $r=a$. This and (33) show that

$$
\int_{V_{r}}\left(a-V^{\sigma}\right) d m \leq C a r
$$

and so (32) gives

$$
\left|\int f d \sigma\right| \leq \frac{C a}{\delta}=C \delta
$$

because $\delta=\sqrt{r}=\sqrt{a}$ by (14).

\section{Proof of Theorem 1.4}

Let, as before, $C_{1}$ be the unit circle. In this proof we shall need to distinguish between a curve as a geometric object and as a parametrized path. If $\gamma: C_{1} \rightarrow$ $\mathbf{R}^{2}$ is a continuous injective mapping, then let $[\gamma]=\left\{\gamma(\xi) \mid \xi \in C_{1}\right\}$ be its image set, which is a Jordan curve. We shall always orient $[\gamma]$ counterclockwise, and for $Z, Z^{\prime} \in[\gamma]$ we shall denote by $[\gamma]_{Z, Z^{\prime}}$ the arc of $[\gamma]$ lying (in the orientation of $[\gamma])$ in between $Z$ and $Z^{\prime}$. Then $[\gamma]_{Z, Z^{\prime}} \cup[\gamma]_{Z^{\prime}, Z}=[\gamma]$

For each $m=0,1, \ldots$ we define an analytic Jordan curve $\left[\gamma_{m}\right]$ and points $Z_{0, m}=\gamma_{m}\left(\xi_{0, m}\right), \ldots, Z_{N_{m}-1, m}=\gamma_{m}\left(\xi_{N_{m}-1, m}\right)\left(\xi_{j, m} \in C_{1}\right)$ on $\left[\gamma_{m}\right]$ in such a way that $\left[\gamma_{m+1}\right]$ lies inside $\left[\gamma_{m}\right]$ except for the points $Z_{0, m}, \ldots, Z_{N_{m}-1, m}$ which lie also on $\left[\gamma_{m+1}\right]$, and we have for all $m$ with some $\rho_{m}>0, \delta_{m} \rightarrow 0$ the properties

(a) $\operatorname{diam}\left(\left[\gamma_{M}\right]_{Z_{j, m}, Z_{j+1, m}}\right)<\delta_{m}$ for all $M \geq m$ and for all $j=0, \ldots, N_{m}-1$,

(b) $\left|\xi_{j, m}-\xi_{j+1, m}\right|<\delta_{m}$ for all $j=0, \ldots, N_{m}-1$,

(c) $\operatorname{dist}\left(\left[\gamma_{M}\right]_{Z_{j, m}, Z_{j+1, m}},\left[\gamma_{M}\right]_{Z_{j+2, m}, Z_{j-1, m}}\right)>\rho_{m}$, for all $M \geq m$ and for all $j=0, \ldots, N_{m}-1$. 
Here the indices are considered modulo $N_{m}$, see below.

Roughly, the Jordan curve $\Gamma$ in the theorem will be the Hausdorff limit of the curves $\gamma_{m}$, but some caution is necessary, since the limit of Jordan curves may not be a Jordan curve.

The construction will be done so that each $Z_{j, m}=\gamma_{m}\left(\xi_{j, m}\right)$ is one of the $Z_{j^{\prime}, m+1}=\gamma_{m+1}\left(\xi_{j^{\prime}, m+1}\right)$, and the parametrization will be such that then $\xi_{j^{\prime}, m+1}=\xi_{j, m}$. In other words, $\gamma_{m}\left(\xi_{j, m}\right)=\gamma_{m+1}\left(\xi_{j, m}\right)$, which implies $\gamma_{M}\left(\xi_{j, m}\right)=$ $\gamma_{m}\left(\xi_{j, m}\right)$ for all $M \geq m$. Thus,

$$
\Gamma(\xi):=\lim _{M \rightarrow \infty} \gamma_{M}(\xi)
$$

exists for all

$$
\xi \in S:=\left\{\xi_{j, m} \mid m=1,2 \ldots, 0 \leq j \leq N_{m}-1\right\} .
$$

By (b) the set $S$ of these numbers is dense in $C_{1}$. Since $\delta_{m} \rightarrow 0$, property (a) shows that $\Gamma$ is uniformly continuous on $S$, so it can be extended to a continuous map from $C_{1}$ into the complex plane. We claim that this extended $\Gamma$ is one-toone on $C_{1}$, hence it defines a Jordan curve. Indeed, if $\xi, \xi^{\prime} \in C_{1}$ are two different points, then, by property (b), there is an $m$ and a $0 \leq j \leq M_{m}-1$ such that $\xi$ lies in between $\xi_{j, m}$ and $\xi_{j+1, m}$ on $C_{1}$, while $\xi^{\prime}$ lies on the arc of $C_{1}$ from $\xi_{j+2, m}$ to $\xi_{j-1, m}$. But then $\Gamma(\xi) \in[\Gamma]_{Z_{j, m}, Z_{j+1, m}}$ while $\Gamma\left(\xi^{\prime}\right) \in[\Gamma]_{Z_{j+2, m}, Z_{j-1, m}}$. By property (c) for all $M \geq m$ the distance of $\left[\gamma_{M}\right]_{Z_{j, m}, Z_{j+1, m}}$ and $\left[\gamma_{M}\right]_{Z_{j+2, m}, Z_{j-1, m}}$ is bigger than $\delta_{m}$. Thus, after taking limits, the distance of $\Gamma(\xi)$ and of $\Gamma\left(\xi^{\prime}\right)$ is at least $\delta_{m}$, so $\Gamma(\xi) \neq \Gamma\left(\xi^{\prime}\right)$.

Clearly, the so obtained Jordan curve $\Gamma$ lies inside every $\gamma_{m}$ (except for the points $\left.Z_{0, m}, \ldots, Z_{N_{m}-1, m}\right)$, and $\Gamma$ contains all the points $Z_{j, m}, m=1,2, \ldots$, $0 \leq j<N_{m}$.

During the construction we shall also have for each $m$ a number $n_{m}$ and a polynomial $Q_{n_{m}, m}(z)=z^{n_{m}}+\cdots$ of degree $n_{m}$ with zeros in the next set $\left\{Z_{0, m+1}, \ldots, Z_{N_{m+1}-1, m+1}\right\}$ such that with $\varepsilon_{m}=1 / 2^{m}$ we have

$$
\left\|Q_{n_{m}, m}\right\|_{\left[\gamma_{m}\right]}<\left(1+\varepsilon_{m}\right) \operatorname{cap}\left(\left[\gamma_{m}\right]\right)^{n_{m}} .
$$

Furthermore, with these $n_{m}$ we shall have, besides (a)-(c) also

(d) $\operatorname{cap}\left(\left[\gamma_{m}\right]\right)^{n_{m}}<\left(1+\varepsilon_{m}\right) \operatorname{cap}\left(\left[\gamma_{m+1}\right]\right)^{n_{m}}$.

The sequence $\left\{n_{m}\right\}$ will be increasing, hence property (d) gives for all $M \geq m$

$$
\operatorname{cap}\left(\left[\gamma_{m}\right]\right)^{n_{m}}<\left(1+\varepsilon_{M-1}\right) \cdots\left(1+\varepsilon_{m}\right) \operatorname{cap}\left(\left[\gamma_{M}\right]\right)^{n_{m}} \leq e^{2 / 2^{m}} \operatorname{cap}\left(\left[\gamma_{M}\right]\right)^{n_{m}},
$$

and upon letting $M \rightarrow \infty$ it follows that

$$
\operatorname{cap}\left(\left[\gamma_{m}\right]\right)^{n_{m}} \leq e^{2 / 2^{m}} \operatorname{cap}([\Gamma])^{n_{m}} .
$$

Thus, in view of (37),

$\left\|Q_{n_{m}, m}\right\|_{[\Gamma]} \leq\left\|Q_{n_{m}, m}\right\|_{\left[\gamma_{m}\right]}<\left(1+\varepsilon_{m}\right) \operatorname{cap}\left(\left[\gamma_{m}\right]\right)^{n_{m}}<\left(1+\varepsilon_{m}\right) e^{2 / 2^{m}} \operatorname{cap}([\Gamma])^{n_{m}}$. 
Since the zeros of $Q_{n_{m}, m}$ lie among the points $Z_{0, m+1}, \ldots, Z_{N_{m+1}-1, m+1}$ which all lie on $\Gamma$, it follows that $\left\{Q_{n_{m}, m}\right\}$ is a sequence of monic polynomials with all their zeros on $\Gamma$ for which

$$
\lim _{m \rightarrow \infty} \frac{\left\|Q_{n_{m}, m}\right\|_{\Gamma}}{\operatorname{cap}(\Gamma)^{n_{m}}}=1
$$

Hence, all what remains is to do the afore-discussed construction with properties (a)-(d). We start from the unit circle $\gamma_{0}=C_{1}$ and with one point on it, $Z_{0,0}=1$, and we are going to do the recursion step $m \rightarrow m+1$ without explicitly showing the index $m$ in $\gamma_{m}, Z_{j, m}$, etc.

Thus, let $[\gamma]$ be an analytic Jordan curve with some (not necessarily analytic) parametrization $\gamma: C_{1} \rightarrow[\gamma]$, and for some $N$ let there be given points $Z_{0}=$ $\gamma\left(\xi_{0}\right), \ldots, Z_{N-1}=\gamma\left(\xi_{N-1}\right)$ on $[\gamma]$. We also set $Z_{j}=Z_{j(\bmod N)}$, i.e. we consider the points $Z_{j}$ modulo $N$ the index $j$, e.g. $Z_{-1}=Z_{N-1}$. We equip $[\gamma]$ with the usual counterclockwise direction. Assume also that there are given positive numbers $\rho, \delta$ such that

(A) $\operatorname{diam}\left([\gamma]_{Z_{j}, Z_{j+1}}\right)<\delta$ for all $j=0, \ldots, N-1$,

(B) $\left|\xi_{i}-\xi_{i+1}\right|<\delta$ for all $j=0, \ldots, N-1$,

(C) $\operatorname{dist}\left([\gamma]_{Z_{j}, Z_{j+1}},[\gamma]_{Z_{j+2}, Z_{j-1}}\right)>\rho$, for all $j=0, \ldots, N-1$.

Let, furthermore, $\varepsilon>0$ be any given positive number.

Since $[\gamma]$ is analytic, the Green's function $g_{\overline{\mathbf{C} \backslash[\gamma]}}:=g_{\overline{\mathbf{C} \backslash[\gamma]}}(\cdot, \infty)$ of the unbounded component of the complement of $[\gamma]$ with pole at infinity has an analytic extension inside $[\gamma]$ to a small neighborhood of $[\gamma]$. Let $\left[\gamma_{\tau}\right]$ be the $g_{\overline{\mathbf{C} \backslash[\gamma]}}(z)=-\tau$ level-curve of this extension, and $\mu_{\left[\gamma_{\tau}\right]}$ be the equilibrium measure of $\left[\gamma_{\tau}\right]$. This latter measure has a smooth (arbitrarily many times differentiable) density with respect to arc measure because $\left[\gamma_{\tau}\right]$ is analytic. For some positive integer $n$ let $I_{1}, \ldots, I_{n}$ be a decomposition of $[\gamma]_{\tau}$ into arcs with $\mu_{\left[\gamma_{\tau}\right]}$-measure equal to $1 / n$, and let

$$
\zeta_{l}=n \int_{I_{l}} t d \mu_{\left[\gamma_{\tau}\right]}(t)
$$

be the center of mass of $\mu_{\left[\gamma_{\tau}\right]}$ on $I_{l}$. It was proved in [15, Theorem 1.4] that for the polynomials

$$
Q_{n}(z)=\prod_{l=1}^{n}\left(z-\zeta_{l}\right)
$$

we have, as $n \rightarrow \infty$,

$$
\left\|Q_{n}\right\|_{[\gamma]}=(1+o(1)) \operatorname{cap}([\gamma])^{n}
$$

where the $o(1)$ is actually geometrically small in $n$ (depending on $\tau$ ). It is clear that no matter how small $\varepsilon>0$ is, for sufficiently small $\tau$ and for sufficiently 
large $n$ all the zeros $\zeta_{l}$ of $Q_{n}$ lie of distance $<\varepsilon / 2$ from $[\gamma]$, and consecutive $\zeta_{l}$ 's on $[\gamma]_{\tau}$ are of distance $<\varepsilon$ from each other. Fix such an $n$ for which

$$
\left\|Q_{n}\right\|_{[\gamma]}<(1+\varepsilon) \operatorname{cap}([\gamma])^{n}
$$

is also true.

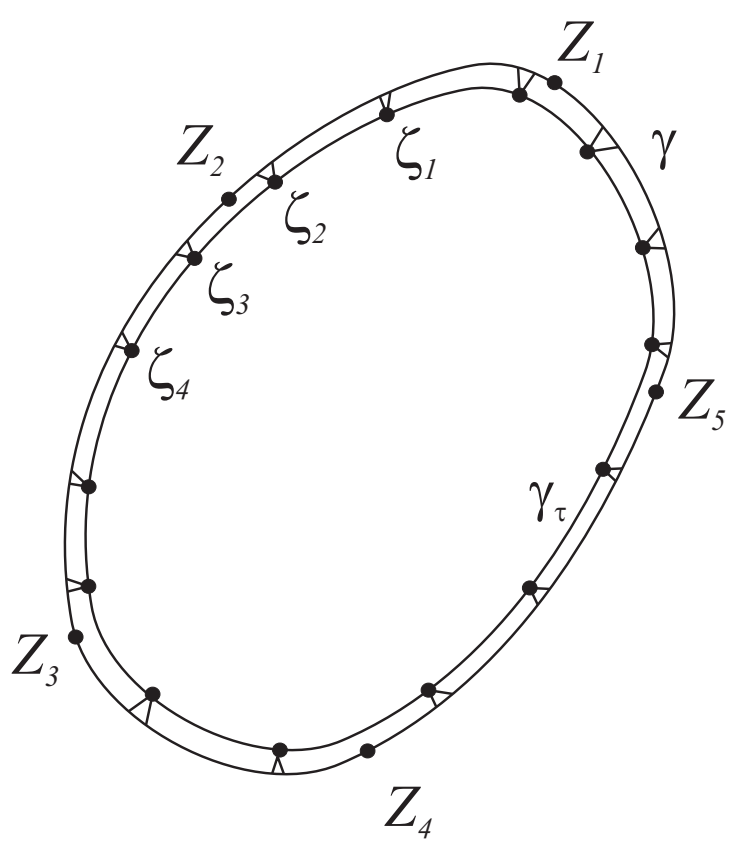

Figure 1: The curves $\gamma, \gamma_{\tau}$ with the points $Z_{j}, \zeta_{j}$ on them and the cuts from $\gamma$ to the points $\zeta_{j}$

Now make appropriate cuts from $[\gamma]$ to each $\zeta_{l}$ depicted in Figure 1 in such a way that the cuts avoid the points $Z_{j}$ and they are made with two segments for each $\zeta_{j}$, and let $[\widehat{\gamma}]$ be the curve obtained this way. Thus, $[\widehat{\gamma}]$ is a Jordan curve lying of distance $<\varepsilon / 2$ from $[\gamma]$ and $[\widehat{\gamma}]$ contains all the previously given points $Z_{0}, \ldots, Z_{N-1}$, as well as the zeros $\zeta_{1}, \ldots, \zeta_{n}$ of $Q_{n}$. It is also clear that we can make the cuts so "narrow" that we have

$$
\operatorname{cap}([\gamma])^{n}<(1+\varepsilon) \operatorname{cap}([\widehat{\gamma}])^{n} .
$$

Now find a $C^{2}$-Jordan curve $[\widetilde{\gamma}]$ that contains all the points $Z_{0}, \ldots, Z_{N-1}$, $\zeta_{1}, \ldots, \zeta_{n}$; except for these points $[\widetilde{\gamma}]$ lies inside $[\widehat{\gamma}]$, and $[\widetilde{\gamma}]$ lies so close to $[\widehat{\gamma}]$ that we have

$$
\operatorname{cap}([\gamma])^{n}<(1+\varepsilon) \operatorname{cap}([\widetilde{\gamma}])^{n}
$$

(cf. (40)), see Figure 2. We may also assume that the curvature of $[\widehat{\gamma}]$ is different from the curvature of $[\gamma]$ at every $Z_{0}, \ldots, Z_{N-1}$ (note that at these points the 


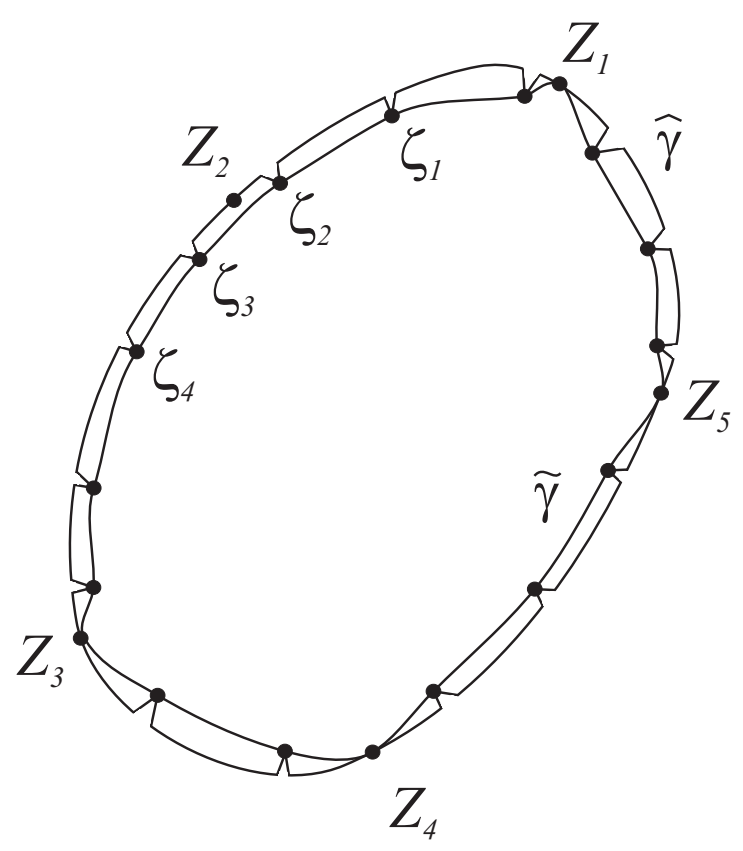

Figure 2: The curves $\widehat{\gamma}$ and $\tilde{\gamma}$; the lemniscate $\sigma$ lies in between of them

curves $[\widehat{\gamma}]$ and $[\gamma]$ touch each other). According to $[7$, Therem 1.1] there is a lemniscate $\sigma$ (i.e. a level set of a polynomial) that is a Jordan curve and lies in between $[\widetilde{\gamma}]$ and $[\widehat{\gamma}]$ except for the common points $Z_{0}, \ldots, Z_{N-1}, \zeta_{1}, \ldots, \zeta_{n}$ which necessarily also lie on $\sigma$. It is clear from (A), (C) above that if $\tau$ is sufficiently small and $n$ is large, furthermore $[\widetilde{\gamma}]$ lies sufficiently close to $[\widehat{\gamma}]$, then we shall have

$$
\operatorname{diam}\left([\sigma]_{Z_{j}, Z_{j+1}}\right)<\delta \quad \text { for all } j=0, \ldots, N-1,
$$

and

$$
\operatorname{dist}\left(\sigma_{Z_{j}, Z_{j+1}}, \sigma_{Z_{j+2}, Z_{j-1}}\right)>\rho \text { for all } j=0, \ldots, N-1 .
$$

We choose a parametrization $\gamma^{*}: C_{1} \rightarrow \sigma$ of $\sigma$ for which $\gamma^{*}\left(\xi_{j}\right)=\gamma\left(\xi_{j}\right)=Z_{j}$ for $j=0, \ldots, N-1$, and if $\zeta_{r}, \ldots, \zeta_{s}$ are the zeros of $Q_{n}$ lying in between $Z_{j}$ and $Z_{j+1}$ on $\sigma(r, s$ depend on $j)$ and

$$
\zeta_{r}=\gamma^{*}\left(t_{r}^{*}\right), \ldots, \zeta_{s}=\gamma^{*}\left(t_{s}^{*}\right),
$$

then the points $t_{r}^{*}, \ldots, t_{s}^{*}$ divide the arc of the unit circle $C_{1}$ lying in between $\xi_{j}$ and $\xi_{j+1}$ into arcs of equal length.

Thus, if $N^{*}=N+n$ and $X_{0}^{*}, \ldots, X_{N^{*}-1}$ are the points $Z_{0}, \ldots, Z_{N-1}$, $\zeta_{1}, \ldots, \zeta_{n}$, then these points lie on the analytic Jordan curve $\left[\gamma^{*}\right]=\sigma$, this curve lies inside $[\gamma]$ except for the points $X_{0}, \ldots, X_{N-1}$ where the two curves $[\gamma]$ and $\left[\gamma^{*}\right]$ touch each other. Furthermore, if the points $X_{0}^{*}, \ldots, X_{N^{*}-1}$ follow 
each other in this order on $\left[\gamma^{*}\right]$, then the distance of consecutive $X_{j}^{*}$ 's is at most $\delta / 2$, and if we set $X_{j}^{*}=\gamma^{*}\left(\xi_{j}^{*}\right)$ with the parametrization $\gamma^{*}$ given above, then consecutive $\xi_{j}^{*}$ 's lie closer than $\delta / 2$ (this follows for large $n$ because the $t_{r}^{*}, \ldots, t_{s}^{*}$ in (44) divide the arc of the unit circle $C_{1}$ lying in between $\xi_{j}$ and $\xi_{j+1}$ into arcs of equal length and for large $n$ the number $s-r$ is large). Furthermore,

$$
\operatorname{dist}\left(\left[\gamma^{*}\right]_{Z_{j}, Z_{j+1}},\left[\gamma^{*}\right]_{Z_{j+2}, Z_{j-1}}\right)>\rho \text { for all } j=0, \ldots, N-1
$$

is also true (see (43)). In view of (41)

$$
\operatorname{cap}([\gamma])^{n}<(1+\varepsilon) \operatorname{cap}\left(\left[\gamma^{*}\right]\right)^{n}
$$

because $\gamma^{*}$ lies outside $[\widetilde{\gamma}]$, and hence its logarithmic capacity is at leas as large as $\operatorname{cap}([\widetilde{\gamma}])$. Setting $\delta^{*}=\delta / 2$ and $2 \rho^{*}$ equal to the minimum of the distances

$$
\operatorname{dist}\left(\left[\gamma^{*}\right]_{Z_{j}^{*}, Z_{j+1}^{*}},\left[\gamma^{*}\right]_{Z_{j+2}^{*}, Z_{j-1}^{*}}\right) \text { for all } j=0, \ldots, N^{*}-1,
$$

we have defined $\gamma^{*}, X_{j}^{*}, j=0, \ldots, N^{*}-1, \delta^{*}, \rho^{*}$ in terms of $\gamma, X_{j}, j=$ $0, \ldots, N-1, \delta, \rho$, and it is clear that for sufficiently small $\tau$ and large $n$ we will have the *-variant of property $(\mathrm{A})$ :

$$
\operatorname{diam}\left(\left[\gamma^{*}\right]_{Z_{j}^{*}, Z_{j+1}^{*}}\right)<\delta^{*} \quad \text { for all } j=0, \ldots, N^{*}-1
$$

By the construction we also have an $n$ and a polynomial $Q_{n}=z^{n}+\cdots$ with zeros in the set $X_{0}^{*}, \ldots, X_{N^{*}-1}$ such that

$$
\left\|Q_{n}\right\|_{[\gamma]}<(1+\varepsilon) \operatorname{cap}([\gamma])^{n}
$$

see $(39)$.

Now all we have to do to make the recursive definition of $\gamma_{m}, X_{j, m}$ etc. is to set $\gamma=\gamma_{m}, X_{j}=X_{j, m}, N=N_{m}, \delta=\delta_{m}, \rho=\rho_{m}$, carry out the previous construction, and set $\gamma_{m+1}=\gamma^{*}, X_{j, m+1}=X_{j}^{*}, N_{m+1}=N^{*}, \delta_{m+1}=\delta^{*}$, $\rho_{m+1}=\rho^{*}$, as well as define $n_{m+1}$ as $n$ and $Q_{n_{m+1}, m+1}$ as $Q_{n}$. Since the $n=n_{m+1}$ can be arbitrarily large, we can select it bigger then the previously constructed $m_{m}$. It is easy to see that all the properties set forth for $\gamma_{m}, X_{j, m}, Q_{n_{m}, m}$ etc. can be satisfied, and the obtained curve $\Gamma$ and formula (38) prove Theorem 1.4.

There is only one point that needs clarification, namely in properties (a) and (c) the assumption is for all $M \geq m$, and not just for $M=m+1$. However, property (a) amounts the same as saying that

$$
\operatorname{diam}\left(\left[\gamma_{m+1}\right]_{Z_{j, k}, Z_{j+1, k}}\right)<\delta_{k}, \quad \text { for all } j=0, \ldots, N_{k}-1
$$

and for all $k \leq m$, and this property is easy to satisfy (exactly as was the $k=m$ case done in (42)) by selecting in the construction $\tau$ small and the curve $[\widetilde{\gamma}]$ close to $[\widehat{\gamma}]$. A similar reasoning can be made regarding property (c). 


\section{Appendix}

In the proof of Theorem 2.2 we used the following facts. Let $0<\alpha<1$ and $\Gamma$ a finite system of $C^{2+\alpha}$-smooth Jordan curves, say of $m$ curves, lying exterior to one another. For some $\tau>0$ consider the set $[-\tau, \tau] \times \Gamma$, and the mapping $H(x, y)=y+\mathbf{n}_{y} x$ from $[-\tau, \tau] \times \Gamma$ onto some subset $V$ of the complex plane, where $\mathbf{n}_{y}$ is the inner unit normal to the exterior domain $\Omega$ to $\Gamma$ at the point $y \in \Gamma$. Then, for small fixed $\tau$,

a) each $\Gamma_{x}:=\left\{y+\mathbf{n}_{y} x \mid y \in \Gamma\right\}, x \in[-\tau, \tau]$, is a union of $m$ Jordan curves

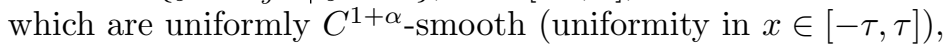

b) the inequality $\left|U^{\mu_{\Gamma_{x}}}(z)+\log \operatorname{cap}\left(\Gamma_{x}\right)\right| \leq C|x|$ is true for all $z \in \Gamma$ with a $C$ that is independent of $z \in \Gamma$ and $x \in[-\tau, \tau]$.

Let $\Gamma_{0}$ be any of the components of $\Gamma$. The $C^{2+\alpha}$-smoothness of $\Gamma_{0}$ means that $\Gamma_{0}$ has a parametrization $\gamma(t)=\gamma_{1}(t)+i \gamma_{2}(t)$, where $\gamma_{1}, \gamma_{2}$ are $2 \pi$ periodic twice continuously differentiable real functions such that $\left|\gamma^{\prime}(t)\right|=$ $\sqrt{\gamma_{1}^{\prime}(t)^{2}+\gamma_{2}^{\prime}(t)^{2}} \neq 0, t \in \mathbf{R}, \gamma(t)$ runs through $\Gamma_{0}$ once in the counterclockwise direction, and there is a constant $C$ such that $\left|\gamma^{\prime \prime}(t)-\gamma^{\prime \prime}(u)\right| \leq C|t-u|^{\alpha}$. Then for $y=\gamma(t) \in \Gamma_{0}$ we have

$$
\mathbf{n}_{y}=\frac{i \gamma^{\prime}(t)}{\left|\gamma^{\prime}(t)\right|}
$$

(note that the unit tangent vector to $\Gamma_{0}$ at $y$ is $\gamma^{\prime}(t) /\left|\gamma^{\prime}(t)\right|$ ), so for any $x$ the function

$$
y+\mathbf{n}_{y} x=\gamma(t)+\frac{i \gamma^{\prime}(t)}{\left|\gamma^{\prime}(t)\right|} x
$$

is $C^{1+\alpha}$-smooth. We claim that for small $|x|$ this function is injective over $t \in[0,2 \pi)$, hence it describes a Jordan curve. Indeed, let $0<a<1$ be a fixed small number. If $0 \leq u<t<2 \pi$, then

$$
\gamma(t)+\frac{i \gamma^{\prime}(t)}{\left|\gamma^{\prime}(t)\right|} x=\gamma(u)+\frac{i \gamma^{\prime}(u)}{\left|\gamma^{\prime}(u)\right|} x
$$

is impossible for $a<t-u<2 \pi-a$ and small $|x|$ (in that case $|\gamma(t)-\gamma(u)| \geq b$ with some $b>0$ that depends only on $\gamma$ and $a$ ). But neither it is possible for $t-u \leq a$ or for $t-u>2 \pi-a$, since otherwise we would have

$$
|x| \frac{i \gamma^{\prime}(t)}{\left|\gamma^{\prime}(t)\right|}-\frac{i \gamma^{\prime}(u)}{\left|\gamma^{\prime}(u)\right|}|=| \gamma(t)-\gamma(u) \mid,
$$

but here the right-hand side is $\geq\left(\min \left|\gamma^{\prime}\right| / 2\right)|t-u|$ if $a$ is sufficiently small, while the left-hand side is at most

$$
\left(\max \left|\left(\frac{\gamma^{\prime}}{\left|\gamma^{\prime}\right|}\right)^{\prime}\right|\right)|t-u||x|,
$$

which smaller than the previous number if $|x|$ is sufficiently small. 
In a similar fashion, it follows that $\gamma(t)+\frac{i \gamma^{\prime}(t)}{\left|\gamma^{\prime}(t)\right|} x$ has non-vanishing derivative, hence it describes a $C^{1+\alpha}$-smooth Jordan curve. This proves part a).

Let $\Gamma_{0, x}$ be the component of $\Gamma_{x}$ lying close to $\Gamma_{0}$, i.e. $\Gamma_{0, x}$ is obtained from $\Gamma_{0}$ as $\Gamma_{x}$ was obtained from $\Gamma$. Let $\Omega_{0, x}$ be the exterior domain to $\Gamma_{0, x}$, $g_{\Omega_{0, x}}(z, \infty)$ the Green's function in $\Omega_{0, x}$ with pole at $\infty$, and $\Psi_{0, x}$ the conformal map from $\Omega_{0, x}$ onto the exterior of the unit circle. Then $g_{\Omega_{0, x}}(z, \infty)=$ $\log \left|\Psi_{0, x}(z)\right|$. Since $\Gamma_{0, x}$ is $C^{1+\alpha}$-smooth, a theorem of Kellogg and Warshawskii (see [8, Theorem 3.6]) tells us that $\Psi_{0, x}(z)$ is also $C^{1+\alpha}$-smooth up to the boundary. As a consequence, $g_{\Omega_{0, x}}(z, \infty)$ is also $C^{1+\alpha}$-smooth up to the boundary $\Gamma_{0, x}$ of $\Omega_{0, x}$. Now (see e.g. [9, Sec. 4.4] or [10, (I.4.8)])

$$
g_{\Omega_{0, x}}(z, \infty)=\log \frac{1}{\operatorname{cap}\left(\Gamma_{0, x}\right)}-U^{\mu_{\Gamma_{0, x}}}(z),
$$

so the right-hand side is again $C^{1+\alpha}$-smooth up to the boundary $\Gamma_{0, x}$. Since the curves $\Gamma_{0, x}$ were uniformly $C^{1+\alpha}$-smooth, the previous conclusion also holds true uniformly in $x \in[-\tau, \tau]$ (with some small $\tau$ ). But the right-hand side in (45) is 0 inside $\Gamma_{0, x}$, hence we obtain

$$
\log \frac{1}{\operatorname{cap}\left(\Gamma_{0, x}\right)}-U^{\mu_{\Gamma_{0, x}}}(z)=g_{\Omega_{0, x}}(z, \infty) \leq C_{0}|x|
$$

for all $z \in \Gamma$ with some $C_{0}$ independent of $x \in[-\tau, \tau]$ (note that the distance from a point $z$ on $\Gamma$ to $\Gamma_{x}$ is at most $\left.|x|\right)$.

After this, let us return to our system of curves $\Gamma_{x}$, and let $\Omega_{x}$ denote their exterior domain. We have again the formula

$$
\log \frac{1}{\operatorname{cap}\left(\Gamma_{x}\right)}-U^{\mu_{\Gamma_{x}}}(z)=g_{\Omega_{x}}(z, \infty) .
$$

Let $\gamma$ be a $C^{1}$-smooth Jordan curve separating $\Gamma_{0, x}$ from the other components of $\Gamma_{x}$ for all $x \in[-\tau, \tau]$. Since $\gamma$ lies of positive distance from all $\Gamma_{x}$, the Green's functions $g_{\Omega_{x}}(z, \infty)$ and $g_{\Omega_{0, x}}(z, \infty)$ all lie in between two positive constants (that are independent of $x \in[-\tau, \tau]$ ) on $\gamma$, hence, by the maximum principle in the ring domain enclosed by $\Gamma_{0, x}$ and $\gamma$, we have

$$
g_{\Omega_{x}}(z, \infty) \leq C_{1} g_{\Omega_{0, x}}(z, \infty)
$$

with some constant $C_{1}$ independent of $x \in[-\tau, \tau]$. Now for $z \in \Gamma$ the difference

$$
\log \frac{1}{\operatorname{cap}\left(\Gamma_{x}\right)}-U^{\mu_{\Gamma_{x}}}(z)
$$

is either 0 (when $x \geq 0$ ) or equals $g_{\Omega_{x}}(z, \infty)$. In either cases

$$
\left|\log \frac{1}{\operatorname{cap}\left(\Gamma_{x}\right)}-U^{\mu_{\Gamma_{x}}}(z)\right| \leq C_{0} C_{1}|x|
$$

is a consequence of (46) and (47). 


\section{References}

[1] V. V. Andrievskii and H-P. Blatt, Discrepancy of signed measures and polynomial approximation, Springer Monographs in Mathematics. SpringerVerlag, New York, 2002.

[2] V. V. Andrievskii and H-P. Blatt, Polynomials with prescribed zeros on an analytic curve, Acta Math. Hungar., 128(2010), 221-238.

[3] D. H. Armitage and S. J. Gardiner, Classical Potential Theory, Springer Verlag, Berlin, Heidelberg, New York, 2002.

[4] J. B. Garnett and D. E. Marshall, Harmonic measure, New Mathematical Monographs 2, Cambridge University Press, Cambridge, 2008.

[5] G. Halász, On the first and second main theorem in Turán's theory of power sums, Studies in pure mathematics, 259-269, Birkhuser, Basel, 1983.

[6] M. Lachance, E. B. Saff and R. S. Varga, Inequalities for polynomials with a prescribed zero, Math. Z., 168(1979), 105-116.

[7] B. Nagy and V. Totik, Sharpening of Hilbert's lemniscate theorem, J. D'Analyse Math., 96(2005), 191-223.

[8] Ch. Pommerenke, Boundary Behavior of Conformal Mappings, Grundlehren der mathematischen Wissenschaften, 299, Springer Verlag, Berlin, Heidelberg New York, 1992.

[9] T. Ransford, Potential Theory in the Complex plane, Cambridge University Press, Cambridge, 1995

[10] E. B. Saff, V. Totik, Logarithmic Potentials with External Fields, Grundlehren der mathematischen Wissenschaften, 316, Springer-Verlag, New York/Berlin, 1997.

[11] V. Totik and P. Varjú, Polynomials with prescribed zeros and small norm, Acta Sci. Math., (Szeged) 73(2007), 593-612.

[12] V. Totik, Polynomials with zeros and small norm on curves, Proc. Amer Math. Soc., 140(2012), 3531-3539.

[13] V. Totik, Asymptotics of Christoffel functions on arcs and curves, Advances in Mathematics, 252(2014), 114-149.

[14] V. Totik, Chebyshev polynomials on compact sets Potential Analysis, 40(2014), 511-524.

[15] V. Totik, Chebyshev polynomials on a systems of curves, J. d'Analyse Math., 118(2012), 317-338. 
[16] J. L. Walsh, Interpolation and Approximation by Rational Functions in the Complex Domain, third edition, Amer. Math. Soc. Colloquium Publications, XX, Amer. Math. Soc., Providence, 1960.

Bolyai Institute

MTA-SZTE Analysis and Stochastics Research Group

University of Szeged

Szeged

Aradi v. tere 1, 6720, Hungary

and

Department of Mathematics and Statistics

University of South Florida

4202 E. Fowler Ave, CMC342

Tampa, FL 33620-5700, USA

totik@mail.usf.edu 